\title{
POBREZA, HAMBRE Y ABIGEATO: OTRAS FORMAS DE RESISTENCIA EN SACATEPÉQUEZ DECIMONÓNICO ${ }^{1}$
}

Jorge Luis Rodríguez Díaz ${ }^{2}$ georgege735@gmail.com

Universidad de San Carlos de Guatemala

\begin{abstract}
Es erróneo creer que las clases sociales oprimidas viven su vida "normal" cuando están resignadas a su suerte por imposibilidad de cambiarla... ya que la violencia manifestada en los momentos críticos se está generando y acumulando todos los días en la entraña de esa existencia "normal"
\end{abstract}

Severo Martínez Peláez

\section{RESUMEN}

En el corazón de la Guatemala rural que nutre a la sociedad colonial con su producción agrícola y a la Corona con sustanciosos tributos, la pobreza genera un aumento en la incidencia de robo de ganado, el cual representa un recurso preciado. El abigeato se vislumbra como una suerte de resistencia por parte de los sectores marginados, que ven violados sus derechos consuetudinarios por el crecimiento de la hacienda y las consecuencias que esta trae a la tradición agrícola local. Esta investigación pone de manifiesto la criminalidad como una forma de resistencia a un sistema opresor y excluyente.

Palabras clave: abigeato, derecho consuetudinario, ganadería, resistencia.

\section{POVERTY, HUNGER AND CATTLE RUSTLING: OTHER FORMS OF RESISTANCE IN NINETEENTH-CENTURY SACATEPÉQUEZ}

\begin{abstract}
In the heart of rural Guatemala that nourished nineteenth-century Colonial society with agricultural production, and provided the Spanish Crown with substantial tribute, poverty generated an increase in cattle rustling. In those times, cattle represented a highly valued resource. In this study, cattle rustling is seen as a form of resistance by the marginalized sectors whose customary rights were violated with the expansion of Colonial farms known as haciendas and the consequences this imprinted on local agricultural. This piece of research evidences criminality as a form of resistance to a system based on oppression and exclusion.
\end{abstract}

Key words: cattle rustling, customary law, cattle raising and resistance.

\footnotetext{
${ }^{1}$ Ponencia presentada en el IX Congreso Centroamericano de Antropología, Guatemala, febrero de 2013.

${ }^{2}$ Estudiante de la Licenciatura en Historia por la Universidad de San Carlos de Guatemala. Líneas de investigación: Pueblos indígenas, administración territorial, control social, justicia y derechos sociales.
} 


\section{INTRODUCCIÓN}

La presente investigación, aunque no pretende agotar el tema de la criminalidad como una forma de resistencia en un contexto de opresión, constituye un acercamiento a las dinámicas de resistencia existentes en la Guatemala del siglo XIX. Está sustentada en función de conocer el desarrollo de la hacienda y sus repercusiones en los pueblos de indios eminentemente agrícolas. La importancia del estudio de la resistencia en el altiplano central y específicamente en San Juan Sacatepéquez radica en la cercanía a la capital de Guatemala, pero también en la función que estos pueblos tuvieron en el complejo sistema de abastecimiento de dicha capital.

En el distrito de San Juan Sacatepéquez para las dos últimas décadas coloniales, salvo raras excepciones como la imposición de nuevas leyes, el aumento de tributos o por abusos cometidos por los indios principales o vecinos españoles, se vislumbra una vida poco tumultuosa, esto a pesar de las exacciones a las que es sometido el pueblo.

La resistencia se aborda desde el enfoque de James Scott, pues aunque tradicionalmente dicho fenómeno ha sido asociado con choque social, conflicto, y momentos críticos como motines y rebeliones, se le encuentra presente en la vida cotidiana y resulta prácticamente imperceptible a la vista de los opresores (Vela 2011:631). La resistencia es característica de sociedades jerarquizadas, en las que el ejercicio del poder permite la extracción de trabajo, bienes, servicios o impuestos a los subordinados, y se crean fricciones entre estos y sus dominadores (Scott 2000:71).

En una sociedad tan jerarquizada, como los dominios coloniales españoles en Indias, conformada por los propios españoles, sus descendientes, indios tributarios y una amplia gama de castas desplazadas, estas contradicciones se hacen patentes en el día a día y de formas diversas: tanto el trabajo semigratuito como las cargas eclesiásticas y el tributo.

En este complejo sistema los tributarios, la población indígena que constituye el sustento económico del sistema se reproduce con base en la agricultura de subsistencia; sin embargo, la opresión genera situaciones de descontento generalizado y desencadena esporádicas protestas; pero cabe preguntarse: ¿Qué sucede en el pueblo en los momentos de supuesta calma? ¿Estaban conformes los subordinados con las injusticias propias del sistema de dominación existente?

En este contexto encontramos formas de resistencia que intentan permanecer en el anonimato, y cuando trascienden a la esfera pública son consideradas delictivas. En varios de estos casos la justificación de los transgresores está basada en la costumbre e incluso legitimada por el derecho. De este modo, el estudio particular de San Juan Sacatepéquez corresponde a la incidencia de casos de abigeato en una región eminentemente indígena y de indudable vocación agrícola más que ganadera.

\section{SUBORDINADOS Y ESTEREOTIPOS}

Según James Scott los subordinados conspiran para confirmar la imagen que los grupos dominantes tienen de ellos, pero también para que esta imagen sea útil a sus propios intereses. De esta cuenta los estereotipos son un recurso y a la vez una forma de opresión para el subordinado (Scott 2000:60), y los grupos dominantes generalmente se sirven de los estereotipos para excluirlos, hacerlos perder sus derechos y condenarlos a recorrer un círculo vicioso que tiene como corolario su aniquilación social.

De este modo, como se observa en San Juan Sacatepéquez, es usual la criminalización de las formas de resistencia, pese a que estas representan un mecanismo de preservación de la propia vida de los involucrados.

El perfil de los trasgresores de San Juan Sacatepéquez puede situarse en un pueblo constituido particularmente por indios tributarios, que llevan sobre sus espaldas el peso de ser la base económica colonial, y condenados a la pobreza y el aislamiento (Martínez 2011:117). Así, nuestros actores no tendrían el perfil de bandoleros sociales en vías de convertirse en héroes, como lo consigna Eric Hobsbawm, y tendrían en común con estos únicamente el odio hacia los explotadores que tratan de imponerse (Hobsbawm 1989:13). Entre los explotadores están las autoridades españolas, pero también los propietarios de las haciendas ganaderas, españoles, que encuentran en los pueblos de indios no solamente tierras para pastura, sino también los brazos necesarios, afectando directamente el microcosmos del pueblo basado en la agricultura. 


\section{GANADERÍA Y CONFLICTO}

A pesar que los pueblos abastecedores de Santiago de Guatemala, antes y después del traslado de 1775, no fueron eminentemente ganaderos (MacLeod 1987:13), podemos hablar de un fuerte aumento en las haciendas en áreas cercanas a los núcleos de los pueblos de indios hacia finales de la colonia. ${ }^{3}$

Las Leyes de Indias regularon la ganadería y restringieron su reproducción a zonas apartadas de dichos poblados. Su idea primordial era privilegiar la producción agrícola - consecuentemente la subsistencia de la población local-sobre la pecuaria.

De esta cuenta se instituye que las haciendas deben estar fuera de los pueblos, puesto que el ganado mayor hace «mucho daño en los maizales de los Indios, especialmente el que anda apartado y sin guarda» y por lo tanto se decretaba «que no se dén estancias ningunas en partes y lugares de donde puedan resultar daños, y no pudiéndose escusar, sean lexos ${ }^{4}$ de los Pueblos de Indios, y sus sementeras». ${ }^{5}$

La ley resulta enfática en la importancia que atribuye al indio como elemento básico de la reproducción económica colonial, al constituir una fuerza de trabajo semigratuita. Asimismo, los recursos ganaderos, no obstante su importancia económica por el alto valor que se les daba, no constituían un elemento esencial de la ingesta indígena, pues solo reducidos sectores del pueblo contaban con capacidad patrimonial para adquirir carne de res, además de que debido a la alta demanda probablemente muchos recursos locales fueron utilizados para el abastecimiento de la capital, que se constituía como el mercado ganadero más importante de la región centroamericana durante la Colonia (Peláez 2007:159).

La hacienda tuvo a finales de dicho periodo un notable crecimiento en zonas adyacentes a pueblos de indios, con la ruralización de españoles y ladinos (Rodas 1999:34), proceso que cobró mayor fuerza por efectos de los terremotos de 1773 y el traslado de la capital, que dispersaron más a la población no

${ }^{3}$ El traslado de la capital supuso pérdidas para algunas familias terratenientes que prefirieron trasladarse a pueblos circunvecinos.

${ }^{4}$ En la transcripción de fuentes primarias preferentemente se mantuvo la grafía de origen, es decir la escritura original.

${ }^{5}$ Recopilación de las Leyes de Indias, Libro IV, titulo XII, ley XII. «Que las estancias para ganados se den apartadas de Pueblos, y sementeras de Indios». indígena a otros centros urbanos y al área rural (Lutz 1994:119, MacLeod 1987:19).

Ya en el siglo XIX se evidencia el aumento de haciendas ganaderas en San Juan Sacatepéquez, como lo comprueban inventarios de bienes y testamentarias, en una de las cuales figuran más de tres haciendas, así como varios activos en ganado y trapiches, hasta conformar un total de 11776 pesos, cifra considerable para aquel momento. ${ }^{6}$

Por otro lado, las quejas por daños de ganado sobre sementeras indígenas pasan de la queja oral a autos judiciales mucho más formales que necesitan ser vistos por jueces de primera instancia. Así, por ejemplo, casos como los de Manuel Pérez, Mateo Boror, y Marcos Pirir, indios que sufrieron daños en sus siembras por un toro de don Manuel Martínez en $1803,{ }^{7}$ no pueden ser verificados por justicias ordinarios, por estar involucrado un español.

\section{EL DELITO DE ABIGEATO}

En términos de la época se considera abigeo o cuatrero a la persona que arreando bestias las desvía con la finalidad de aprovecharlas (Escriche 1851:14). Según la ley corresponde la pena capital de muerte como castigo; sin embargo, Escriche afirma que esta pena cayó en desuso por ser considerada demasiado severa, y fue sustituida por trabajos obligatorios.

En el caso de San Juan Sacatepéquez resulta importante evidenciar la recurrencia de los casos de abigeato, especialmente de ganado vacuno, pues según podemos observar es una forma de resistencia de sectores menesterosos, que se ven perjudicados por la presión que los nuevos grupos ejercen sobre la tierra. Este problema es fundamental, siendo que la tierra es el medio de subsistencia familiar indígena, pero también indispensable para cubrir los compromisos económicos con la Corona y la Iglesia, es decir, el tributo y el diezmo.

El primero, que inicialmente se podía pagar en especie, a partir de 1747 empezó a recaudarse en dinero, asimismo para inicios del siglo XIX se aumentó

\footnotetext{
${ }^{6}$ Archivo General de Centroamérica, en adelante AGCA, A.1, legajo 2986, expediente 28411. «Doña Paula Argueta sobre demanda a la testamentaria de su padre Don José Argueta, contra sus albaceas».

${ }^{7}$ AGCA, A.1, Leg. 2951, exp. 27824, 14 de abril de 1803. «Manuel Perez Mateo Boror, y Marcos Pirir naturales de San Juan sobre los perjuicios que sufren en sus sementeras de los ganados de Don Manuel Martines».
} 
de un promedio de 11 a 16 reales, es decir, 2 pesos (Martínez 2011:55), motivo por el cual la producción agrícola tradicional -maíz y frijol particularmente- no era suficiente, y muchos se veían precisados de ocuparse en otras labores, generalmente mal remuneradas, para cubrir sus obligaciones.

Cabe preguntarse como lo haría Scott ¿Cuáles eran las motivaciones de los campesinos? (Scott 2000:223), ¿mataban las reses para calmar el hambre, por placer o porque querían cobrarse los daños que estas causaban a sus cosechas? Según esta teoría podrían ser correctas todas estas razones, pues debemos observar lo que representa el ganado para los sujetos en cuestión.

Los tributarios mantenían la costumbre, observada en las Leyes de Indias, de que el abuso por parte de las bestias sobre cosechas no se tolerara, debido a que una de las normas dictaba que las estancias de ganado mayor no debían estar dentro de legua y media de las reducciones antiguas y el doble de las nuevas; la ley enunciaba: «todos los dueños le tengan con buena guarda, pena de pagar el daño, que hizieren: y los Indios puedan matar el ganado, que entrare en su tierra sin pena alguna». ${ }^{8}$

\section{RESISTENCIA MATERIAL Y SIMBÓLICA EN PUEBLOS DE INDIOS}

En términos teóricos podemos observar en Sacatepéquez algunos elementos que forman parte de la resistencia de los grupos oprimidos según la teorización de Scott.

\section{DIGNIDAD Y NECESIDAD DE LA INFRAPOLÍTICA}

Los sistemas de dominación generan cotidianamente insultos y ofensas a la dignidad humana: la apropiación del trabajo, los castigos públicos, y la denigración generan expresiones de cólera y violencia por parte de los subordinados, la cual debe ser disimulada por el bien propio y de la familia (Scott 2000:63).

La infrapolitica, como le llama James Scott, es precisamente este conjunto de formas de operar que se constituyen en el cimiento de una acción política

\footnotetext{
${ }^{8}$ Recopilación de las Leyes de Indias, Libro VI, título III, ley XX. «Que cerca de las reducciones no haya estancias de ganado».
}

más compleja que no podría existir sin ella en las complicadas condiciones en que viven los oprimidos. ${ }^{9}$ Por lo tanto las formas elementales de la infrapolítica permanecen como una defensa a fondo de los desvalidos (Scott 2000:236).

Se da en espacios sociales determinados y entre grupos de actores particulares. No utiliza solamente el lenguaje, sino una amplia gama de prácticas y estratagemas discretas que tienen la función de minimizar la apropiación que los opresores hacen sobre sus recursos (Scott 2000:38, 222).

En San Juan, tributarios explotados se valen de la costumbre para justificar sus actuaciones: es el caso de Manuel Pérez, Mateo Boror, y Marcos Pirir en 1803, quienes al ver dañadas sus siembras por un toro de don Manuel Martínez hicieron uso de la tradición: mataron al animal, y aunque su dueño solicita que los indios cerquen las sementeras, su petición es negada por la autoridad, y en este caso se defiende a los indios afectados.

De esta forma la misma tradición era una salvaguarda para las acciones de los mencionados tributarios. Evidentemente esta norma dejó paulatinamente de ser práctica, cuando el pueblo de San Juan Sacatepéquez se vio afectado por el traslado de españoles, pero también de ladinos que encontraban precisamente en las haciendas de estos un espacio ideal para su reproducción.

Las repercusiones se dieron en pleno siglo XIX, cuando la norma fue paulatinamente abandonada debido principalmente a la importancia que se le asignó a la propiedad privada y a los derechos de los nuevos grupos sociales en pueblos de indios, ${ }^{10} \mathrm{con}$ la violación constante que se hizo sobre los derechos de estos últimos.

\footnotetext{
${ }^{9}$ Martínez Peláez afirma que la opresión colonial utilizada para obtener del indio un máximo de riqueza, implicó un fracaso permanente, y una prolongada frustración de muchos aspectos de su desarrollo humano como consecuencia de la pobreza, el aislamiento cultural y el bloqueo ideológico. De esta cuenta afirma que percibiendo sus propias limitaciones frente a un enemigo más poderoso tiene como resultado el temor imperante.

${ }^{10}$ Indudablemente para el siglo XIX ya era un hecho consumado la presencia de población no indígena en los pueblos de indios, que aunque tenía prohibido morar en dichos pueblos, fue paulatinamente aceptada por la imposibilidad de la corona de alternativas para esta población. Véase también MacLeod, Murdo, citado en la bibliografía.
} 


\section{LAS REDES INFORMALES}

Debido a que la actividad política explícita está casi prohibida, la resistencia se reduce a las redes informales de la familia, los vecinos, los amigos y la comunidad, en vez de adquirir una organización formal (Scott 2000:236), siendo esta la estructura que permite la protección de esta resistencia.

Así, en el auto judicial de junio de 1814 contra Lázaro Chajón, Santiago Chamalé, Cipriano Boror, y Lorenzo Chamalé se consignaba que en el rancho de Chamalé:

[...] se havian matado ya tres reses, que los cueros y la Carne [estaban escondidos] en la Milpa [...] Lazaro Chajo [...] había vendido un cuero á Cruz Saban en dos y medio [reales], y haviendo llegado con la justicia al reconocimiento se halló ser cierto estaba en poder de Saban [...] que las hermanas del preso Pedro Boror se save han llebado á vender Carne al Pueblo de San Pedro y pansas a este. ${ }^{11}$

Es necesario mencionar que estas actividades estaban estrictamente proscritas, debido a que el expendio de carne competía a una sola persona que tenía adjudicada su distribución en el pueblo. ${ }^{12}$

Finalmente, en su declaración Lázaro Chajón acepta su culpabilidad, sin embargo, excusando algunos de los cargos:

[...] el Savado diez y ocho del pasado junio, como á las nueve de la noche estando cuidando su milpa que esta inmediata al rancho de Lorenzo Chamalé vió llegar á su milpa una res, y que en esto gritó llamando a Santiago Chamalé; para que le ayudase á corretear la dicha res, y que luego llegó santilago Chamalé; y después Cipriano Boror, y juntos todos tres corretearon la res, que conocieron que era una ternera de Don Fernando, ó de su yerno Don Rafael Banses, que la corieron de la milpa dos ocaciones, y á la tercera ocacion que volbió se determinaron á cojerla con un lazo de persoga que Santiago sacó de su rancho, y haviendola lazado el que declara la mataron, y se compartieron de la Carne todos $\operatorname{tres}^{13}[\ldots]$ que es cierto que le vendió el cuero a

${ }^{11}$ AGCA, A.1, leg. 2981, exp. 28307. 21 de junio de 1814. «Contra Lazaro Chajon Santiago Chamalec Cipriano Boror, y Lorenzo Camalec por rovo de ganado».

${ }^{12}$ A.1, Leg. 2988, Exp. 28436. 1814. «Representacion de este común de Naturales sobre que se remate la Carniseria de este Pueblo a quien mas de y Providencia dictada por la superioridad».

${ }^{13}$ Ibíd. Julio 1 de 1814. Declaración de Lázaro Chajón.
Cruz Saban, que no se acuerda si en real y medio, ó mas, y que este cuero se lo dio regalado Julián Canandes moso de la Labor de Don Fernando Davila, porque lo encontró en el campo, y le dijo que aquella Baca que alla estaba muerta, y se la estaban comiendo lo sopes, había muerto de mal parto, y que entonces le pidió el cuero a dicho Julian, y el se lo quitó a la Baca muerta, y se lo trajo a su casa a secarlo, y luego se lo vendió a Cruz Saban. ${ }^{14}$

Nuevamente se evidencia la utilización de estas estrategias para salvaguardar los intereses de los grupos en cuestión, y de esta manera evitar las consecuencias que conlleva la transgresión de la ley.

\section{RESISTENCIA, DELITO Y CASTIGO}

Evidentemente, existe una línea divisoria muy delgada entre la resistencia y el delito, de este modo la violación pública a los límites impuestos es un estímulo para que otros hagan lo mismo poniendo en riesgo el sistema imperante (Martínez 2011:114). Por lo tanto, la afirmación del territorio simbólico se da a través de castigos públicos que hacen que otros pierdan el ánimo de intentar cualquier desafío abierto (Scott 2000:232).

En casos de abigeato se observa reiteradamente que las penas más comunes son prisión, además de cargos económicos que van desde el pago del valor de las reses hasta embargos, en casos en que los culpables no cuenten con medios para hacer pagos.

En el caso de Vicente Pocón y Lucas Chim, los cuales son acusados del robo de un ternero en la labor de Castellanos, al insistírseles en que debían pagar el valor del ternero expresaban que ya habían pagado en el grillete en que les había puesto el juez preventivo por algunos días. ${ }^{15}$

Resulta ilustrativo el caso de Juan Osla, pues a pesar de no ser abigeato sino simplemente robo de aves, es condenado junto a su hermano y presenta queja por abuso de la fuerza por parte de los justicias, ${ }^{16}$ quienes habrían dado tres docenas de azotes a su hermano y veinticinco a él, con lo cual se confir-

\footnotetext{
${ }^{14}$ Idem.

${ }^{15}$ AGCA, A.1, leg. 2958, exp. 27970. «Contra los indios de San Juan Zacatepequez Francisco Juarez, Vizente Pocon y Jacinto Sequem por ladrones de ganado en las labores de Don Fernando Dávila, D. Manuel Martínez y Sebastian Castellanos».

${ }^{16}$ AGCA, A.1, leg. 2958, exp. 27949. Diciembre de 1806. «Contra Juan Osla y sus dos hermanos yndios de San Juan Sacat. Por Rovos rateros de gallinas y chumpipes».
} 
ma la brutalidad de algunos castigos que formaban parte ya del día a día.

[...] hago a V. S. presente que mi hermano Lazaro Pazan, se halla gravemente malo, de una asotada que le dieron las Justicias de dicho mi pueblo, que hasta fue menester que el padre lo holehase no habiendo mas motivo: que Bentura Mosin y Tomasa Boch, me levantaron falsamente que yo y mi hermano Lazaro Pasan, habíamos tobado tres chompipes $[\ldots] .^{17}$

\section{REFLEXIONES FINALES}

La opresión del sistema colonial y la apropiación que dicho sistema hace de los recursos de los subordinados se constituye en factor esencial de desigualdad, y genera como consecuencia pobreza moral y económica en la mayoría de población indígena.

El sistema colonial no debe considerarse como opresor de la generalidad indígena, pues es necesario hacer distinción de «los principales» que se constituyen como intermediarios entre el régimen y los tributarios, pues tienen los beneficios correspondientes a dicha posición, y en ellos se observan posibilidades de mejores condiciones de vida.

Los indios oprimidos al verse a merced de la desigualdad, reaccionan de manera violenta, sin embargo esta violencia debe ser evitada por el bien individual y familiar. De esta manera no se observan motines o alzamientos relevantes en el pueblo de San Juan Sacatepéquez para las dos últimas décadas coloniales.

Formas de resistencia como el hurto, la evasión, producción y comercialización clandestina de bienes, conforman estrategias utilizadas por indios, pero también por otras castas desposeídas en San Juan Sacatepéquez para reapropiarse algunos de los recursos de que fueron expoliados.

Los casos de abigeato que trascienden como causas criminales en San Juan Sacatepéquez, y en los pueblos de indios en general, son solamente una muestra de los que se daban con regularidad, muchos de los cuales jamás fueron castigados debido al desconocimiento de los afectados.

Las transgresiones indígenas al orden establecido en San Juan Sacatepéquez, llevadas a cabo por medio de la resistencia simbólica y material, tienen como consecuencia el castigo que se constituye como elemento simbólico que evitará que dichas transgresiones sean emuladas por las mayorías. La reproducción de dichas transgresiones puede llegar a constituirse como elemento desestabilizador del orden establecido.

\section{FUENTES DE CONSULTA}

Escriche, Joaquín, 1851, Diccionario Razonado de Legislación y Jurisprudencia, Librería de Rosa, Bouret y Cia, París.

Hobsbawm, Eric, 1989, Rebeldes primitivos. Estudio sobre las formas arcaicas de los movimientos sociales en los siglos XIX y XX, Ariel, Barcelona.

Lutz, Christopher, 1994, «Evolución demográfica de la población ladina», en Historia General de Guatemala, tomo II. Sociedad Económica de Amigos del País, Guatemala.

MacLeod, Murdo, 1987, Relaciones Étnicas y la Sociedad Indígena en la Provincia de Guatemala, 1620-1800, Seminario de Integración Social Guatemalteca, Guatemala.

Martínez Peláez, Severo, 2011, Motines de Indios. La Violencia Colonial en Centroamérica y Chiapas, F\&G Editores, Guatemala.

Peláez Almengor, Oscar Guillermo, 2007, La Ciudad Ilustrada, Centro de Estudios Urbanos y Regionales-Universidad de San Carlos de Guatemala, Guatemala.

Rodas, Isabel, 1999, «Identidad, asentamiento y relaciones de parentesco de los españoles de Patzicía (Siglos XVI-XVIII)», en T. Little-Siebold y J. Piel (eds.), Entre Comunidad y Nación: La historia de Guatemala vista desde lo local y lo regional, CIRMA, Guatemala.

Scott, James, 2000, Los Dominados y el Arte de la Resistencia. Discursos Ocultos, Era, México.

Vela Castañeda, Manolo, 2011, Guatemala, la infinita historia de las resistencias, Secretaría de la Paz de la Presidencia de la República, Guatemala.

Fecha de recepción: 5 de septiembre de 2013.

Fecha de aceptación: 25 de noviembre de 2013.

${ }^{17}$ Idem. 